\title{
GPU Acceleration of the Locally Selfconsistent Multiple Scattering Code for First Principles Calculation of the Ground State and Statistical Physics of Materials ${ }^{\text {tr }}$
}

\author{
Markus Eisenbach ${ }^{\mathrm{a}, *}$, Jeff Larkin ${ }^{\mathrm{b}}$, Justin Lutjens ${ }^{\mathrm{b}}$, Steven Rennich ${ }^{\mathrm{b}}$, James \\ H. Rogers $^{\mathrm{a}}$ \\ ${ }^{a}$ Oak Ridge National Laboratory, Oak Ridge, TN 37831, USA \\ ${ }^{b}$ NVIDIA Corporation, Santa Clara, CA 95050, USA
}

\begin{abstract}
The Locally Self-consistent Multiple Scattering (LSMS) code solves the first principles Density Functional theory Kohn-Sham equation for a wide range of materials with a special focus on metals, alloys and metallic nano-structures. It has traditionally exhibited near perfect scalability on massively parallel high performance computer architectures. We present our efforts to exploit GPUs to accelerate the LSMS code to enable first principles calculations of $\mathrm{O}(100,000)$ atoms and statistical physics sampling of finite temperature properties. We reimplement the scattering matrix calculation for GPUs with a block matrix inversion algorithm that only uses accelerator memory. Using the Cray XK7 system Titan at the Oak Ridge Leadership Computing Facility we achieve a sustained performance of $14.5 \mathrm{PFlop} / \mathrm{s}$ and a speedup of 8.6 compared to the CPU only code.
\end{abstract}

Keywords: First-Principles, Monte-Carlo, Phase Transitions PACS: 05.10.Ln, 75.40.Mg, 64.70.kd

\section{Introduction}

Density Functional Theory (DFT)[5], especially in the Kohn-Sham formulation[8] represents a major, well established, methodology for investigating materials

\footnotetext{
This manuscript has been authored by UT-Battelle, LLC under Contract No. DEAC05-00OR22725 with the U.S. Department of Energy. The United States Government retains and the publisher, by accepting the article for publication, acknowledges that the United States Government retains a non-exclusive, paid-up, irrevocable, world-wide license to publish or reproduce the published form of this manuscript, or allow others to do so, for United States Government purposes. The Department of Energy will provide public access to these results of federally sponsored research in accordance with the DOE Public Access Plan (http://energy.gov/downloads/doe-public-access-plan).

* Corresponding author

Email address: eisenbachm@ornl.gov (Markus Eisenbach)
} 
from first principles, that enables the efficient calculation of the energy and other properties in solid state systems. Most computational approaches to solving the Kohn-Sham equation for electrons in materials attempt to solve the eigenvalue problem for periodic systems directly. The solution of the eigenvalue problem for dense matrices results in cubic scaling in the system size. Additionally these spectral methods require approximations such as the use of pseudopotentials or linearized basis sets for all electron methods to make the size of the basis set manageable.

In this paper we utilize a different approach to solving the Kohn-Sham equation using multiple scattering theory in real space. The basis of this method is formed by the Kohn-Korringa-Rostocker (KKR) method [9, 7], that allows for the solution of the all electron DFT equations without the need for linearisation.

The multiple scattering approach to solving the Kohn-Sham equation avoids the explicit diagonalization of the Hamiltonian to obtain the Kohn-Sham orbitals. Instead the method calculates the Green's function $G\left(\vec{r}, \vec{r}^{\prime} ; E\right)$ using the regular $\left(Z_{L}^{n}(\vec{r} ; E)\right.$ and irregular $J_{L}^{n}(\vec{r} ; E)$ solutions of the Schrödinger equation for the single site scatter $n$ with the single site scattering matrix $t_{n}$. [1] This results in the multiple scattering Green's function in eq. 1 connecting points $\vec{r}_{n}$ and $\vec{r}_{m}$ at sites $n$ and $m$ respectively.

$$
\begin{aligned}
G\left(\vec{r}_{n}, \vec{r}_{m}^{\prime} ; E\right)= & \sum_{L L^{\prime}} Z_{L}^{n}\left(\vec{r}_{n} ; E\right) \tau_{n m}^{L L^{\prime}}(E) Z_{L^{\prime}}^{m}\left(\vec{r}_{m}^{\prime} ; E\right) \\
& -\sum_{L} Z_{L}^{n}\left(\vec{r}_{<} ; E\right) J_{L}^{n}\left(\vec{r}_{>} ; E\right) \delta_{n m}
\end{aligned}
$$

with the scattering path matrix

$$
\tau=\left[I-t G_{0}\right]^{-1} t .
$$

With this Green's function the charge and magnetisation densities can be readily calculated by integrating to the Fermi energy:

$$
\begin{aligned}
& n(\vec{r})=\frac{1}{\pi} \operatorname{Im} \int^{E_{F}} \operatorname{Tr} G(\vec{r}, \vec{r} ; E) \mathrm{d} E \\
& \vec{m}(\vec{r})=\frac{1}{\pi} \operatorname{Im} \int^{E_{F}} \operatorname{Tr} \vec{\sigma} G(\vec{r}, \vec{r} ; E) \mathrm{d} E
\end{aligned}
$$

In the following section we explain the details for the calculation of $\tau$ wich is the computationally most demanding part of the calculation.

\subsection{The LSMS Algorithm}

To solve the Kohn-Sham equations arising in the context of DFT, we use a real space implementation of the multiple scattering formalism that was outlined above. The real space treatment of DFT allows the formulation of computational approaches that scale linearly in the system size. One such approach is the Locally Self-consistent Multiple Scattering (LSMS) method, that calcu-

lates the electronic properties from first principles in real space, but introduces 
some approximations that make the treatment of large systems possible. The LSMS method is based on the observation that good convergence of material properties can be obtained by solving the Kohn-Sham equation of density functional theory at a given atomic site by considering not the whole system, but only a sufficiently large neighbourhood, the local interaction zone (LIZ), of each site. The details of this method for calculating the Green function and the total ground state energy $E[n(\vec{r}), \vec{m}(\vec{r})]$ are described elsewhere [17, 4]. Linear scaling is achieved by limiting the scattering distance of electrons in the solution of the multiple scattering problem. Additionally the LSMS code allows the constraint of the magnetic moment directions [13] which enables the sampling of the excited magnetic states in the Wang-Landau procedure described below. The main difference of LSMS from other KKR formulations lies in the evaluation of the scattering path matrix $\tau$ (eq. 2). The details of this calculation are described in the next sections. The implementation of the scattering matrix for GPUs represents the main work we present here to achieve the acceleration of the LSMS code.

\section{Scattering Matrix Calculation}

\subsection{Construction of the m-Matrix}

To calculate the $\tau$-matrix (eq. 2) the first step involves constructing the scattering matrix

$$
m=I-t G_{0}
$$

to be inverted, where $I$ is the unit matrix, $t$ is the vector of all single site scattering matrices inside the LIZ and $G_{0}$ is the matrix of structure constants describing the non-scattering propagation of electrons inside the LIZ. The $m$ matrix is constructed from blocks that are associated with the sites $i$ and $j$ in the local interaction zone.

$$
m_{i j}=I \delta_{i j}-t_{i} G_{0}^{i j}
$$

Each of these block can in principle be evaluated in parallel. The structure constants are given by the expression

$$
G_{0, L L^{\prime}}^{i j}(E)=4 \pi i^{l-l^{\prime}} \sum_{L^{\prime \prime}} C_{L^{\prime} L^{\prime \prime}}^{L} D_{L^{\prime \prime}}^{i j}(E)
$$

where $L$ are the combined $(l, m)$ indices and $C_{L^{\prime} L^{\prime \prime}}^{L}$ are the Gaunt coefficients. The factor $D_{L^{\prime \prime}}^{i j}(E)$ is given by the following equation.

$$
D_{L}^{i j}(E)=-i^{l+1} \sqrt{E} h_{l}\left(\sqrt{E} R_{i j}\right) Y_{L}^{*}\left(\hat{R}_{i j}\right)
$$

Here $h_{l}(x)$ are the spherical Hankel functions and $Y_{L}(\hat{r})$ are the spherical harmonics. 


\subsection{Matrix Inversion}

The most computationally intensive part of the LSMS calculation is the matrix inversion to obtain the multiple scattering matrix $\tau$. (eq. 2) The amount of computational effort can be reduced by utilising the fact that for each local interaction zone only the left upper block $\left(\tau_{00}\right)$ of the scattering path matrix $\tau$ is required. The size of this matrix is determined by the $l_{\max }$ cutoff and for a non-collinear calculation the size of $\tau_{00}$ is $2\left(l_{\max }+1\right)^{2} \times 2\left(l_{\max }+1\right)^{2}$. LSMS uses an algorithm that reduces the amount of work needed while providing excellent performance due to its reliance on dense matrix-matrix multiplications that are available in highly optimized form in vendor or third party provided implementations (i.e ZGEMM in the BLAS library).

The method employed in LSMS to calculate the required block of the inverse relies on the well known expression for writing the invers of a matrix in term of inverses and products of subblocks:

$$
\left(\begin{array}{cc}
A & B \\
C & D
\end{array}\right)^{-1}=\left(\begin{array}{cc}
U & V \\
W & Y
\end{array}\right)
$$

where

$$
U=\left(A-B D^{-1} C\right)^{-1}
$$

and similar expressions for $V, W$, and $Y$. This method can be applied multiple times to the subblock $U$ until the desired block $\tau_{00}$ of the scattering path matrix is obtained. The diagonal blocks $A$ and $D$ are square matrices of sizes $k \times k$ and $k^{\prime} \times k^{\prime}$ respectively, whereas $B$ and $C$ are in general rectangular matrices of size $k \times k^{\prime}$ and $k^{\prime} \times k$.

This allows us to formulate an algorithm for the block inverse $\tau_{00}$ that is used in LSMS as outlined in alg. 1. In this algorithm we select a sub-block $D$ of size $k_{i} \times k_{i}$ and use the expression $A-B D^{-1} C$ in eq. 10 to reduce the size of the matrix to be inverted by $k_{i}$. This procedure can be applied repeatedly until the final block that remains to be inverted has size $k \times k$ where $k=2\left(l_{\max }+1\right)^{2}$. The inversion of this final block will result in the desired result for $\tau_{00}$.

\subsection{Implementation Details}

For the present discussion it is important to note that the computationally most intensive part is the calculation of the scattering path matrix $\tau$ for each atom in the system by inverting the multiple scattering matrix. While the size of the scattering path matrix $\tau$ is proportional to the number of sites in the local interaction zone and to $\left(l_{\max }+1\right)^{2}$ (typically a few thousand, e.g. for $l_{\max }=3$ and 113 atoms in the local interaction zone, the rank is 3616.), the only a small diagonal block of $\tau$ (typically $32 \times 32$ ) will be required in the subsequent calculations of site diagonal observables, that only depend on $\vec{r}$ and $\vec{r}^{\prime}$ inside the same atomic volume $n$ in the expression for the Green's function in eq. 1 (i.e. magnetic moments, charge densities, and total energy). This allows us to employ the algorithm described in the previous section for maximum utilisation of the on node floating point compute capabilities. 


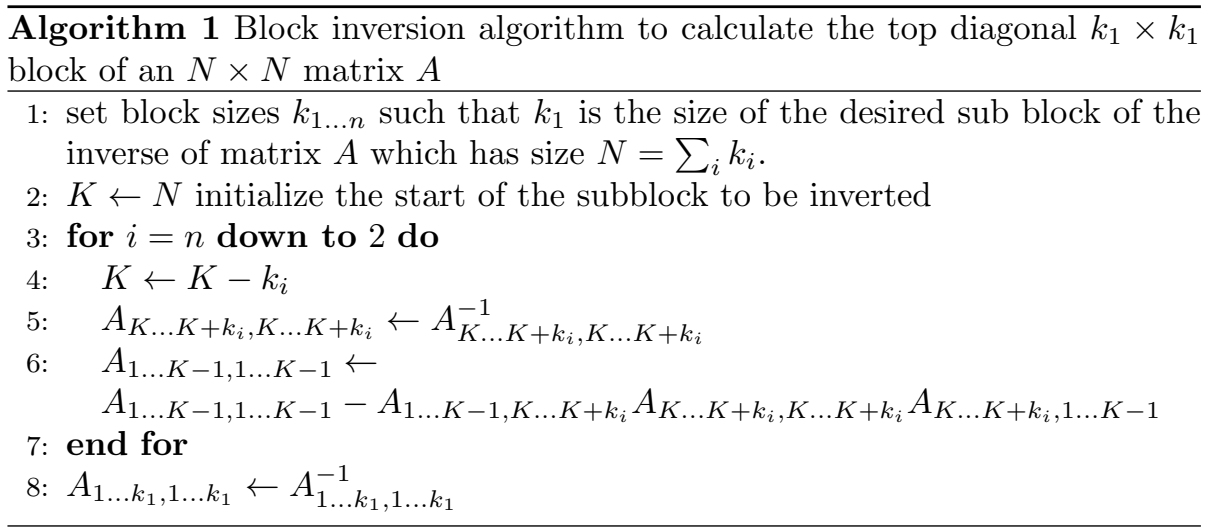

From benchmarking a typical calculation of 1024 iron atoms with a local interaction zone radius of $12.5 a_{0}$ on the AMD processors on Titan, we find that the majority of time $(95 \%)$ and floating point operations are spent inside the inversion of the multiple scattering matrix to obtain the $\tau$-matrix. About half of the remaining time is used to construct this matrix. Thus our approach to accelerate the LSMS code for GPUs concentrated on these two routines that will be presented in detail in the previous two sections.

To construct the scattering matrix (eq. 5, the size of these individual atom blocks is given by the cut-off in the $l$ expansion of the scattering expansion. For a spin-canted calculation the size of a block is $2\left(l_{\max }+1\right)^{2}$, i.e. for a typical $l_{\max }=3$ each of the $m_{i j}$ blocks has rank 32. The indices inside each block label the angular momentum $l, m$. For a typical number of $O(100)$ atoms in the local interaction zone, there are $O(10,000)$ blocks of the $m$ matrix that need to be calculated, thus providing significant parallelism that can be exploited on accelerators. On the accelerator the $m$ matrix is first initialized to a unit matrix to account for the $I \delta_{i j}$ part. The single site scattering matrices are currently calculated on the CPU, as this involves only the numerical evaluation of ordinary differential equations for initial values determined by the energy and the angular momentum $l$ and are communicated as needed to remote nodes and transferred to the GPU memory.

The structure constants $G_{0, L L^{\prime}}^{i j}(E)$ are geometry dependent and the atomic distances $R_{i j}$ and directions $\hat{R}_{i j}$ are transferred to the GPU memory at the beginning of the program and remain there unchanged.

These structure constants are evaluated inside a CUDA kernel that is parallelized in $L, L^{\prime}$ and executed in multiple streams for $i$ and $j$.

The matrix inversion algorithm (Alg. 1 ) needed to obtain $\tau_{00}$ requires matrix multiplications and inversion of the diagonal subblocks. For the matrix multiplication on the GPUs we can exploit the optimized version of these routines that are readily available in the cuBLAS library. The size of the intermediate block sizes used in the matrix inversion serves as a tuning parameter to optimize the performance of our block inversion algorithm. As the block size becomes 


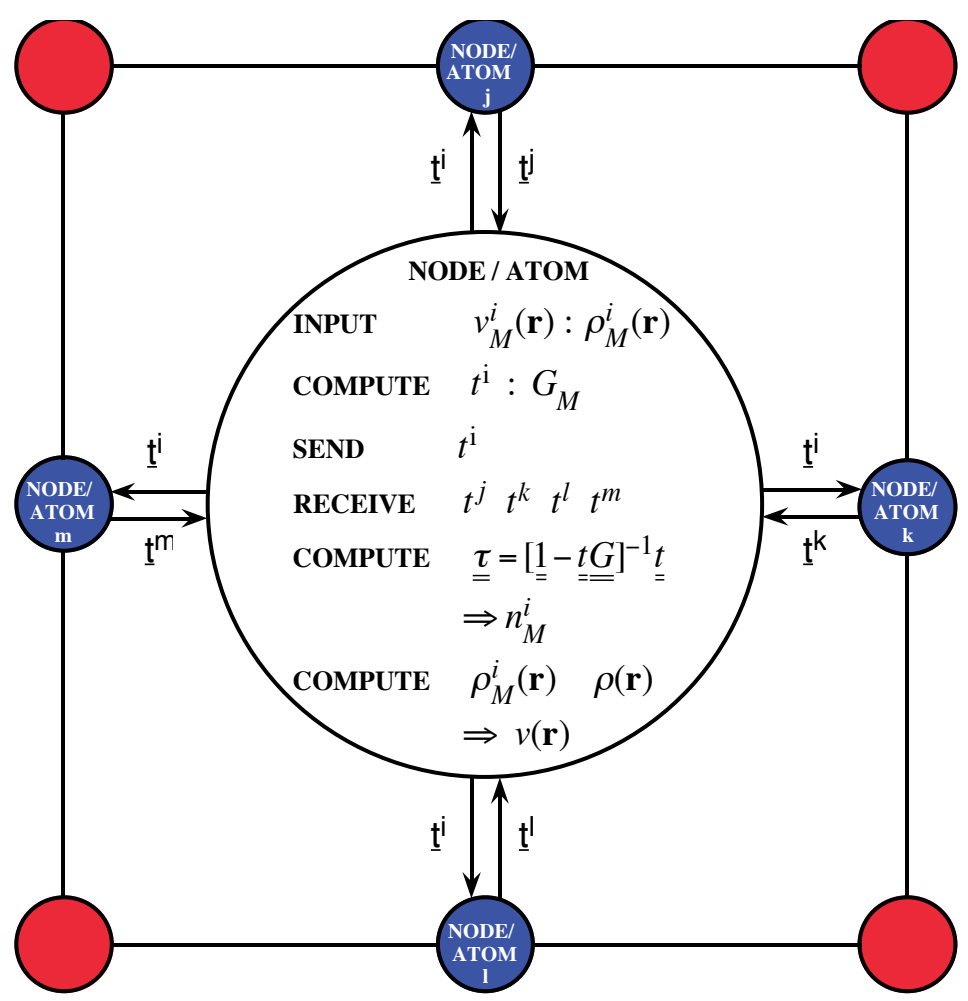

Figure 1: Parallelization scheme of the LSMS method.[17]

larger, the resulting matrices entering the matrix multiplication usually result in significant improvements of the matrix multiplication performance at the cost of performing more floating-point operations then are strictly needed to obtain the final $\tau_{00}$ block. Thus there exists a optimum intermediate block size that minimizes the runtime of the block inversion. For the CPU only code this is achieve at a rank of the intermediate blocks of approximately 1000. For the GPU version we employ an optimized matrix inversion algorithm written in CUDA that executes the whole inversion in a single kernel in GPU memory, thus avoiding costly memory transfers and kernel launches. The maximal rank of double complex matrices that can be handled by this algorithm is 175 , thus providing the size limit for the intermediate blocks and the block size for which we observe the maximum performance of the GPU version reported in this paper. For the matrices that appear in the LSMS method we did not observe numerical problems associated with this approach and the final results obtained are identical for a full inversion of the $m$ matrix and the block inversion described above both for the large block sizes used on CPUs and the smaller sizes required for the GPU accelerated version. We expect that future generations of accelerators that allow the on-device inversion of larger matrices will allow 
further improvements of the acceleration of the GPU based code.

The final product $t_{i} G_{i j}$ is evaluated using batched cuBLAS double complex matrix multiplications.

\section{Wang-Landau Monte-Carlo sampling}

The LSMS method allows the calculation of energies for a set of parameters or constraints $\left\{\xi_{i}\right\}$ that specify a state of the system that is not the global ground state. Examples of this include arbitrary orientations of the magnetic moments or chemical occupations of the lattice sites. Thus we can calculate the energy $E\left(\left\{\xi_{i}\right\}\right)$ associated with these sets of parameters. Evaluating the partition function

$$
Z(\beta)=\sum_{\left\{\xi_{i}\right\}} e^{-\beta E\left(\left\{\xi_{i}\right\}\right)},
$$

where $\beta=1 / k_{B} T$ is the inverse temperature and the sum is over all possible configurations $\left\{\xi_{i}\right\}$, allows the investigation of the statistical physics of the system and the evaluation of its finite temperature properties. In all but the smallest most simple systems (e.g. for a few Ising spins), it is computational intractable to perform this summation directly. Monte-Carlo methods have been used successfully to evaluate these very high dimensional sums or integrals using statistical importance sampling. The most widely used method is the Metropolis method [10], which generates samples in phase space with a probability that is given by the Boltzmann factor $e^{-\beta E\left(\left\{\xi_{i}\right\}\right)}$.

For our work we have chosen to employ the Wang-Landau Monte-Carlo method [16, 15], which is a method to calculate the density of states $g(E)$ of the system for the phase space spanned by the set of classical parameters that describe the system.

We have parallelized the Wang-Landau procedure by employing multiple, parallel walkers that update the same histogram and density of states [3]. The WL-LSMS code uses a hybrid parallelization scheme. At the top level, the code parallelizes over concurrent random walkers, where we use a master-slave scheme, with a master that accumulates the density of states of the system, and the slaves that execute the random walks, each running its own instance of the LSMS method. The master executes the Wang-Landau algorithm as shown in alg. 2. The second parallelization level is the LSMS portion of the code, where domain decomposition is used with one atom per processing core. In typical production runs, the WL method would use a hundred to a few thousand concurrent walkers, and the LSMS portion would be parallelized over up to a few thousand processing cores. The method hence will scale to hundred thousand or millions of processing cores. The schematics of the parallelization structure are shown in fig. 2.

\section{Scaling and Performance}

The WL-LSMS code has been known for its performance on scalability, as has been shown on CPU only architectures, such as the previous Jaguar system at 


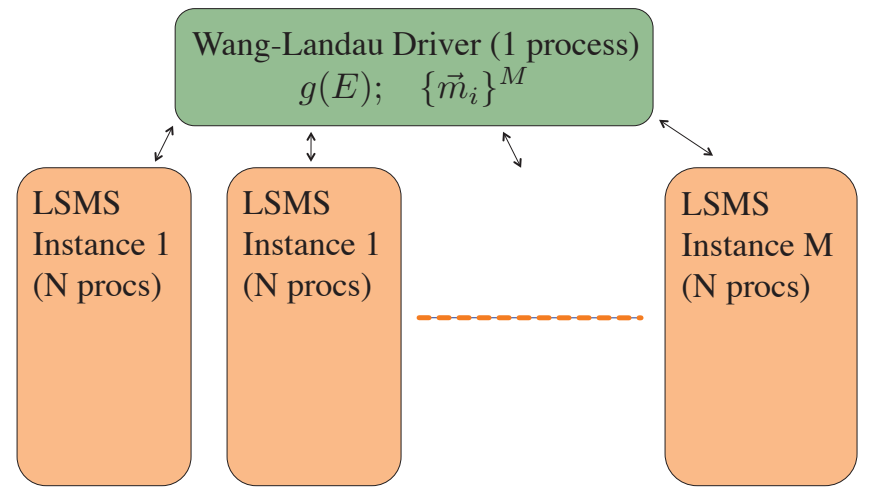

Figure 2: Parallelization strategy of the combined Wang-Landau/LSMS algorithm. The Wang-Landau process generates random configurations $\left\{\xi_{i}\right\}$ for $M$ walkers and updates a single density of states $g(E)$. The energies for these $N$ atom systems are calculated by independent LSMS processes. This results in two levels of communication, between the WangLandau driver and the LSMS instances, and the internal communication inside the individual LSMS instances spanning $N$ processes each.

the Oak Ridge Leadership Computing Facility [3]. The acceleration of significant portions of the code for GPUs, combined with major restructuring of the high level structure of the LSMS code was able to maintain the excellent scalability of the code. In fig. 3 we show the near perfect weak scaling of the LSMS code in the number of atom, while maintaining the number of atom per compute node over five orders of magnitude from 16 iron atoms to 65,536 atoms, while achieving a speedup factor of 8.6 for the largest systems compared to using the CPUs only on the Titan system at Oak Ridge. This performance puts calculations of million atom size systems within reach for the next generation of supercomputers such as the planned Summit system at the Oak Ridge Leadership Computing Facility.

For the statistical sampling with the Wang-Landau method as described above, we tested the scaling of the code in the number of walkers. The performance tests were done for 1024 iron atoms and the energies were self-consistently calculated for a LIZ radius of $12.5 a_{0}$ allowing us to achieve a sampling rate of nearly one Monte-Carlo sample per second on Titan. To limit the runtime for the scaling tests we executed five Wang-Landau Monte-Carlo steps per walker this will incur higher initialization overhead when compared with typical production runs that will typically execute 30-50 or more Monte-Carlo steps per walker and run. The scaling of the energy samples per wall-time is shown inf fig. 4. The results shown are provided in units of the total number of MonteCarlo steps generated per unit wall-time, thus directly relating to the runtime for a simulation run.

To assess the improvements in the computational and power efficiency that resulted from the porting of the significant portions of the code to GPU acceler- 


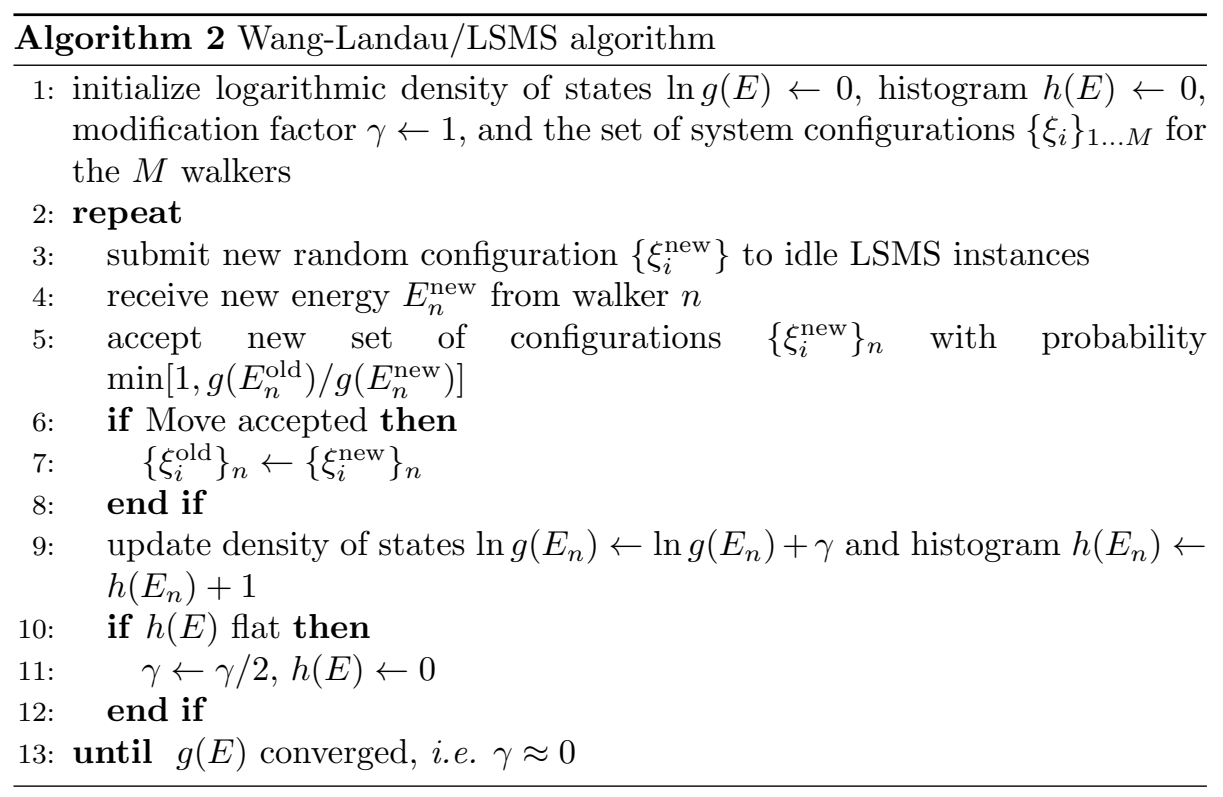

ators, we have run an identical WL-LSMS calculation for 1024 iron atoms with 290 walkers on 18561 nodes on Titan at the Oak Ridge Leadership Computing Facility for 20 Monte-Carlo steps per walker. We measured the instantaneous power consumption at the power supply to the compute cabinets which includes the power for compute, memory and communication as well as line losses and the secondary cooling system inside the cabinets, but excludes the power consumption of the file system and the chilled water supply. The measurements were performed both for a CPU only run as well a for a computation utilizing the GPUs. The results are shown in fig. 5. The difference in power consumption between the compute intensive LSMS calculations, that take most of the time, and Monte-Carlo steps that are marked by a significant drop in the power consumption is obvious and this allows a clear comparison of the two runs.

\section{Applications}

In this section we review results that we have obtained using the method described in this paper to calculating the Curie temperatures of various materials and ordering transitions in alloys. In particular we have applied this method to magnetism in iron and cementite [2] and in $\mathrm{Ni}_{2} \mathrm{MnGa}[11]$. Additionally we calculated the order - disorder transition temperature in $\mathrm{CuZn}[6]$.

For the WL-LSMS calculations of the magnetic transitions, the atoms are placed on lattices with lattice parameters corresponding to the experimental room temperature values. The self-consistently converged potentials for the ferromagnetic or ferrimagnetic ground states were used for all the individual frozen-potential energy calculations in the combined Wang-Landau/LSMS al- 


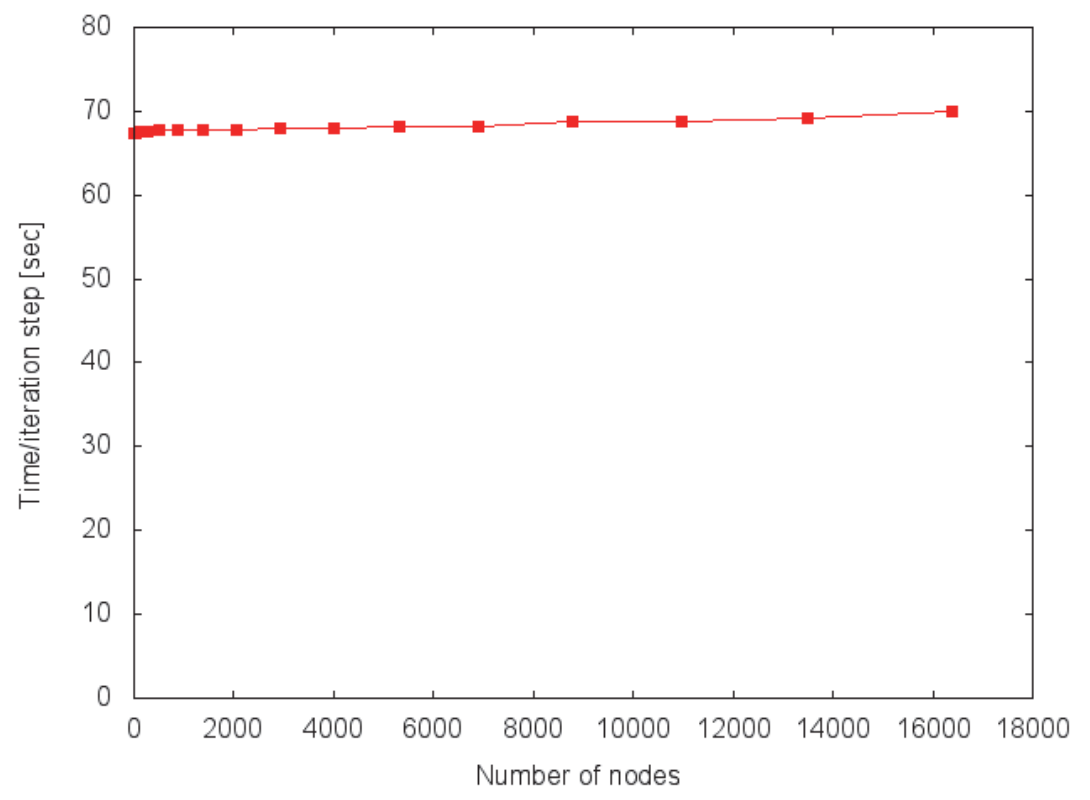

Figure 3: Weak scaling of LSMS on Titan utilizing the GPU accelerators for a bulk iron calculation. 16 atoms on 4 nodes require $67.343 \mathrm{sec}$ per iteration step and 65536 atoms on 16384 nodes require $69.988 \mathrm{sec}$, resulting in a parallel scaling efficiency of $96 \%$ across Titan.

gorithm. The calculations were performed by randomly choosing a site in the supercell and randomly picking a new moment direction. In the case of Fe and $\mathrm{Fe}_{3} \mathrm{C}$ the convergence criterion for the Wang-Landau density of states was chosen to be the convergence of Curie temperature. The density of state thus obtained was used to calculate the specific heat. The peak in the specific heat allows us to identify the Curie temperature to be $980 \mathrm{~K}$ for iron, in good agreement with the experimental value of $1050 \mathrm{~K}$. The Curie temperature obtained for $\mathrm{Fe}_{3} \mathrm{C}$ is $425 \mathrm{~K}$ which again is in good agreement with the experimental value of $480 \mathrm{~K}$. [2] For $\mathrm{Ni}_{2} \mathrm{MnGa}$ the Curie temperature reported is $185 \mathrm{~K}$, well below the experimental value of $351 \mathrm{~K}$.[11] The small cell used in these calculation (144 atoms) will have resulted in a significant finite size error. Additionally, it is known that the localized moment picture that underpins our WL-LSMS calculations does miss important contributions to the fluctuations that determine the finite temperature magnetism in nickel, which will contribute to the reduction of the calculated Curie temperature from the experimental value. This was already observed by Staunton et al. [12] in disordered local moment calculations that underestimate the Curie temperature of pure $\mathrm{Ni}$ and they find $450 \mathrm{~K}$ as opposed to the experimental value of $631 \mathrm{~K}$. We propose to include fluctuations in the magnitude of local moments. Preliminary calculations with a Heisenberg model that is extended with the inclusion of the magnitude of the local moment as a variable indicates that this can result in an increase in the Curie temperature. 


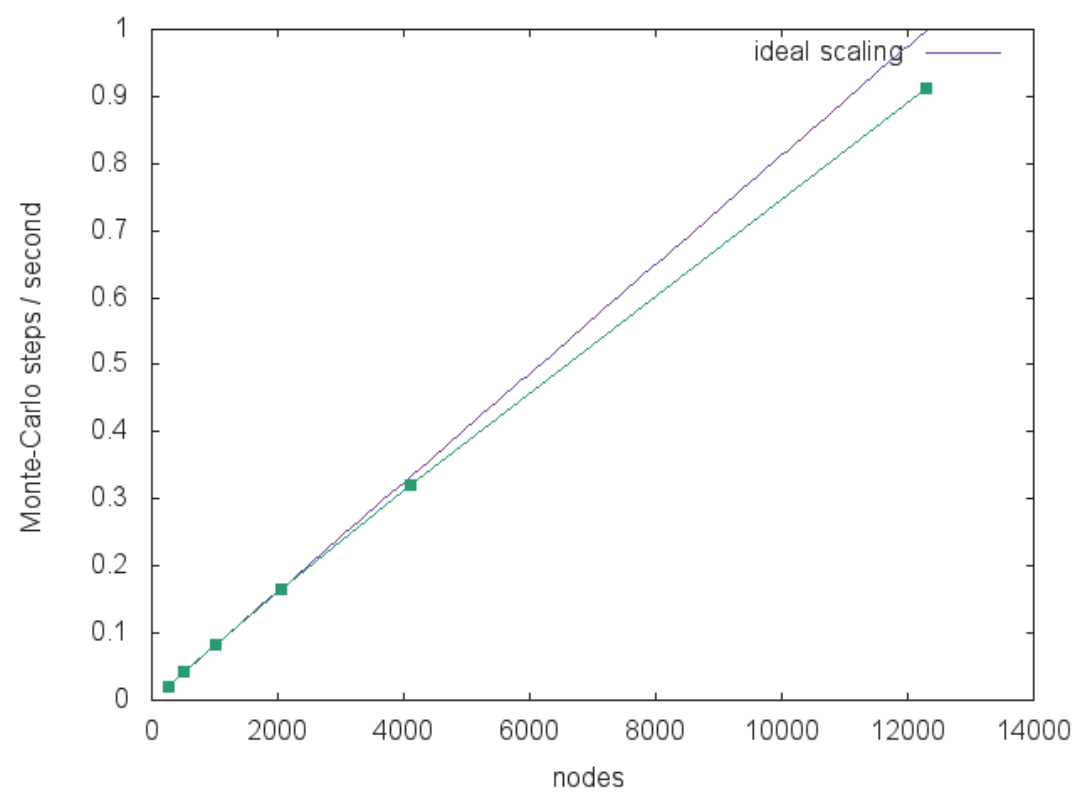

Figure 4: Scaling of Wang Landau LSMS on Titan for 1024 Fe calculations, using 128 nodes per Monte-Carlo walker. The code shows good scaling in the number of states sampled with increasing number of walkers. With two walkers on 257 nodes the code generates 0.0208 samples/second and with 96 walkers on 12289 nodes 0.9128 samples/second are generated, thus achieving a parallel efficiency of $92 \%$.

For the calculation of the order-disorder transition in CuZn, the atoms are placed on bcc lattice with a lattice constant corresponding to the high temperature disorder phase. The simulation cell contains a total of 250 atoms (125 $\mathrm{Cu}$ and $125 \mathrm{Zn}$ atoms). The calculation employed Monte Carlo moves that exchange pairs of atoms, such preserving the atomic concentration of the system. Since every move changes the local chemical environment of the atoms involved, a fully selfconsistent energy calculation was required at each Wang-Landau step. Neglecting lattice vibrations, this calculation find the ordering transition from a high temperature $\mathrm{A} 2$ (bcc) phase to a low temperature $\mathrm{B} 2(\mathrm{CsCl})$ structure at $870 \mathrm{~K}$, which is in reasonable agreement with the experimentally observed temperature of $750 \mathrm{~K} .[6]$

\section{Future Developments}

As Monte-Carlo methods require many energy calculations to reach convergence in the thermodynamic quantities of interest, any improvements in the underlying sampling method will need to be exploited. Future computer architectures will require much higher degrees of parallelism, which could be achieved by increasing the number of Monte-Carlo walkers in the Wang-Landau method described above. A problem of this paralleization scheme for large numbers of 


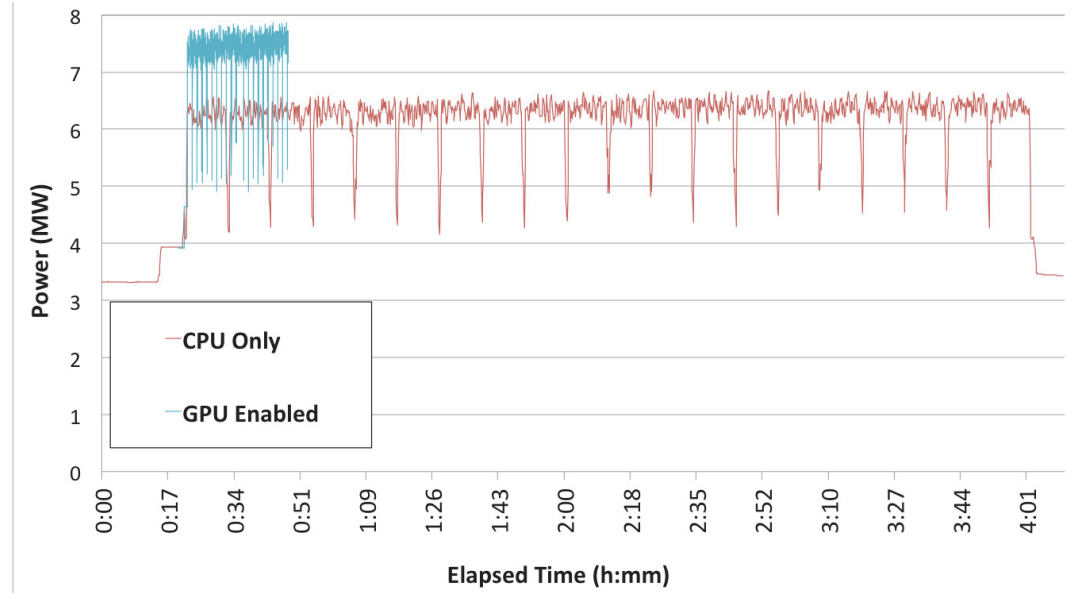

Figure 5: Power consumption traces for identical WL-LSMS runs with 1024 Fe atoms on 18,561 Titan nodes (99\% of Titan). 14.5 PF sustained vs 1.86 PF CPU only. Runtime is $8.6 \mathrm{X}$ faster for the accelerated code, Energy consumed is 7.3X less. GPU accelerated code consumed 3,500 kW-hr, CPU only code consumed 25,700 kW-hr.

walkers is a significant convergence slow-down as walkers get trapped in low probability areas.[18] To overcome this problem, we are exploring the ReplicaExchange Wang-Landau method inside WL-LSMS, which has been shown to achieve superlinear speedup for some model systems [14]. As the ReplicaExchange Wang-Landau method does not rely on a master-slave approach, we expect better scalability in preparation for exascale systems. Also, this sampling method's improved efficiency, will enable us to perform calculations for significantly increased phase spaces, sampling additional degrees of freedom.

The multiple scattering formalism currently implemented in LSMS 3 assumes spherical atomic scattering potentials and a constant interstitial potential between the atomic sites. ("Muffin-Tin approximation") To be able to relax lattice structures, perform first-principles molecular dynamics or to include lattice vibration within statistical physics calculations, it is necessary to go beyond this approximation and consider space filling non-spherical scattering potentials ("Full potential"). While the solution of the ordinary differential equations for single site scattering currently require minimal computing resources, the differential equations for full potential are significantly more expensive to solve, approaching the cost of the matrix inversion for typical local interaction zone sizes. Consequently it will be important to port these full potential scattering solvers to GPUs in future versions of the code.

\section{Conclusions}

We have shown that for some classes of calculations, it is possible to make efficient use of GPU accelerators with a reasonable amount of code modification 
work. The acceleration of the code additionally results in significant energy savings while maintaining its scalability. Consequently the code and work presented in this paper enables the first principles investigation of materials at scales that were previously hard to access and pushes the possibilities for first principles statistical physics. Ongoing work involves extending the capabilities of LSMS presented in this paper to non spherical atomic potentials and to solving the Dirac equation for the electrons in solids, which will allow the first principles investigation of the coupling of magnetic and atomic degrees of freedom and other effects involving atomic displacements. These additions to the code will require additional work to accelerate the single site solvers for GPUs as in these cases

a significant amount of compute resources will be needed to solve the single site equation for non spherical scatterers.

\section{Acknowledgements}

This work has been sponsored by the U.S. Department of Energy, Office of Science, Basic Energy Sciences, Material Sciences and Engineering Division (basic theory and applications) and by the Office of Advanced Scientific Computing (software optimization and performance measurements). This research used resources of the Oak Ridge Leadership Computing Facility, which is supported by the Office of Science of the U.S. Department of Energy under contract no. DE-AC05-00OR22725.

[1] Ebert, H., Ködderitzsch, D., Minár, J.: Calculating condensed matter properties using the KKR-Green's function method-recent developments and applications. Reports on Progress in Physics 74, 096501 (2011)

[2] Eisenbach, M., Nicholson, D.M., Rusanu, A., Brown, G.: First principles calculation of finite temperature magnetism in $\mathrm{Fe}$ and $\mathrm{Fe} 3 \mathrm{C}$. Journal of Applied Physics 109(7), 07E138 (2011)

[3] Eisenbach, M., Zhou, C.G., Nicholson, D.M., Brown, G., Larkin, J., Schulthess, T.C.: A scalable method for ab initio computation of free energies in nanoscale systems. In: Proceedings of the Conference on High Performance Computing Networking, Storage and Analysis. pp. 64:1-64:8. SC '09, ACM, New York, NY, USA (2009)

[4] Eisenbach, M., Györffy, B.L., Stocks, G.M., Újfalussy, B.: Magnetic anisotropy of monoatomic iron chains embedded in copper. Phys. Rev. B 65, 144424 (Mar 2002)

[5] Hohenberg, P., Kohn, W.: Inhomogeneous electron gas. Phys. Rev. 136, B864-B871 (Nov 1964)

[6] Khan, S.N., Eisenbach, M.: Density-functional Monte-Carlo simulation of CuZn order-disorder transition. Phys. Rev. B 93(2), 024203 (Jan 2016), http://link.aps.org/doi/10.1103/PhysRevB.93.024203 
[7] Kohn, W., Rostoker, N.: Solution of the Schrödinger equation in periodic lattices with an application to metallic Lithium. Phys. Rev. 94, 1111-1120 (Jun 1954), http://link.aps.org/doi/10.1103/PhysRev.94.1111

[8] Kohn, W., Sham, L.J.: Self-consistent equations including exchange and correlation effects. Phys. Rev. 140, A1133-A1138 (Nov 1965)

[9] Korringa, J.: On the calculation of the energy of a Bloch wave in a metal. Physica 13, 392-400 (Aug 1947)

[10] Metropolis, N., Rosenbluth, A.W., Rosenbluth, M.N., Teller, A.H., Teller, E.: Equation of state calculations by fast computing machines. Journal of Chemical Physics 21, 1087 (1953)

[11] Nicholson, D.M., Odbadrakh, K., Rusanu, A., Eisenbach, M., Brown, G., Evans III, B.M.: First principles approach to the magneto caloric effect: Application to $\mathrm{Ni}_{2} \mathrm{MnGa}$. Journal of Applied Physics 109(7), 07 A942 (2011)

[12] Staunton, J., Gyorffy, B.: Onsager cavity fields in itinerant-electron paramagnets. Phys. Rev. Lett. 69, 371-374 (Jul 1992)

[13] Stocks, G.M., Eisenbach, M., Újfalussy, B., Lazarovits, B., Szunyogh, L., Weinberger, P.: On calculating the magnetic state of nanostructures. Progress in Materials Science 52(2-3), 371 - 387 (2007)

[14] Vogel, T., Li, Y.W., Wüst, T., Landau, D.P.: Generic, hierarchical framework for massively parallel wang-landau sampling. Phys. Rev. Lett. 110, 210603 (May 2013)

[15] Wang, F., Landau, D.P.: Determining the density of states for classical statistical models: A random walk algorithm to produce a flat histogram. Phys. Rev. E 64, 056101 (2001)

[16] Wang, F., Landau, D.P.: Efficient, multiple-range random walk algorithm to calculate the density of states. Phys. Rev. Lett. 86(10), 2050-2053 (2001)

[17] Wang, Y., Stocks, G.M., Shelton, W.A., Nicholson, D.M.C., Temmerman, W.M., Szotek, Z.: Order-N multiple scattering approach to electronic structure calculations. Phys. Rev. Lett. 75, 2867 (1995)

[18] Yin, J., Landau, D.: Massively parallel Wang-Landau sampling on multiple GPUs. Computer Physics Communications 183(8), 1568 - 1573 (2012), http://www.sciencedirect.com/science/article/pii/S0010465512000859 